\title{
Original Research \\ Malawian impressions of expatriate physicians: A qualitative study
}

\author{
Natasha Parekh ${ }^{1}$, Adam P. Sawatsky' ${ }^{2}$ Ihunanya Mbata ${ }^{3}$, Adamson S. Muula ${ }^{4}$, Thuy Bui ${ }^{1}$ \\ 1. Division of General Internal Medicine, University of Pittsburgh Medical Center, Pittsburgh, Pennsylvania, USA \\ 2. Division of General Internal Medicine, Mayo Clinic, Rochester, Minnesota, USA \\ 3. TeamHealth, Oak Ridge Methodist Medical Center, Oak Ridge, Tennessee, USA \\ 4. School of Public Health and Family Medicine, College of Medicine, University of Malawi, Blantyre, Malawi
}

Correspondence to: Natasha Parekh (parekhn@upmc.edu)

\section{Background}

\section{Abstract}

In many low-income countries, including Malawi, expatriate physicians serve diverse roles in clinical care, education, mentorship, and research. A significant proportion of physicians from high-income countries have global health experience. Despite the well-known benefits of global health experiences for expatriates, little is known about local physician and trainee impressions of their expatriate counterparts. The objective of this study was to explore University of Malawi College of Medicine (COM) physicians' and trainees' impressions of expatriate physicians.

Methods

We conducted a cross-sectional qualitative study using semi-structured interviews with COM medical students, interns, registrars, and faculty. Through open coding, we developed a codebook that we applied to interview transcripts and used thematic analysis to identify major themes.

Results

We interviewed 46 participants from across the continuum of medical education at two teaching hospitals in Malawi. Participants discussed themes within the following domains: perceived benefits of expatriate physicians in Malawi, perceived challenges, past contributions, and perceived roles that expatriate physicians should play going forward. Malawian faculty and trainees appreciated the approachability, perspectives, and contribution to education that expatriates have provided, though at times some have been perceived as aggressive, unable to relate to patients and trainees, deficient at adapting to the setting, and self-serving. Potential roles that Malawian physicians and trainees feel expatriates should serve include education, training, capacity building, and facilitating exchange opportunities for local physicians and trainees.

Conclusions

This study highlights the perceived benefits and challenges that physicians and trainees at the COM have experienced with their expatriate counterparts, and suggests roles that expatriates should play while abroad. These findings can be used to help inform existing global health guidelines, assist with the establishment of host institution expectations for global health programmes, and guide individual expatriate physicians who hope to optimise their roles abroad.

\section{Introduction}

Expatriate physicians have taken on diverse roles in underserved settings globally, including the provision of clinical care, research, education, and mentorship. They practice in a variety of settings and work through formal international aid organisations, universities, as well as independently. A significant proportion of trainees and physicians from high-income countries participate in global health activities: in the United States (US), 31\% of medical students participate in international experiences, over 50 residency programmes offer global health tracks and international rotations, and over 6000 medical mission trips originate from the US annually. ${ }^{1-3}$ These experiences are positive from the perspective of expatriates and their native countries: physicians with global health experiences are more likely to work with underserved populations and engage in community service opportunities. ${ }^{4-6}$ Reported benefits include gaining experience with diverse pathologies, learning to work with limited resources, developing clinical and surgical skills, participating in education, and experiencing new cultures.

In low-income settings, expatriate physicians serve important roles in addressing physician shortages and helping to establish medical education infrastructure. ${ }^{8,9}$ In Malawi specifically, expatriate physicians held half of the faculty positions at the University of Malawi College of Medicine (COM) in 2007 and have been integral in developing COM postgraduate training programmes. ${ }^{8,10,11}$ In the last decade, http://dx.doi.org/10.4314/mmj.v28i2.3
Malawian physicians have increased their representation in the faculty, but because of staff shortages, the COM still depends on expatriates to fill academic positions. ${ }^{11}$

Despite ongoing expatriate workforce needs and the notable benefits of global health experiences for expatriate physicians, little is known about the influence of these experiences on host institutions, physicians, and trainees. In order to ensure mutual and reciprocal benefits of global health experiences for both host institutions and expatriates, it is imperative to investigate hosts' perspectives, expectations, and needs. We therefore sought to explore these themes at the COM.

\section{Methods}

We conducted a qualitative study using in-depth, semistructured interviews to explore attitudes towards expatriate physicians and their ideal roles at the COM in 2014. For the study's purposes, an expatriate is a person who resides temporarily or permanently in a country other than his or her country of origin. ${ }^{12}$ This study was approved by the University of Malawi College of Medicine Research and Ethics Committee (COMREC) and institutional review boards at the University of Pittsburgh and the Mayo Clinic.

\section{Setting and participants}

The COM opened in 1991 and has graduated 250 medical doctors. ${ }^{13,14}$ From 2006 to 2014 , the graduating class increased from 15 to 56 students, with plans for continued growth. ${ }^{13,15}$ The COM offers postgraduate Master of Medicine degrees in internal medicine, paediatrics, general surgery, 
ophthalmology, orthopaedics, obstetrics and gynaecology, psychiatry, anaesthesia and intensive care, nephrology, and emergency medicine. ${ }^{11,14}$

We identified our initial interview candidates through mixed sampling methods; we used criterion sampling among COM fourth-year medical students with clinical experience, and we approached interns, registrars, and faculty from across a breadth of specialties to obtain broad perspectives. We then used snowball sampling and enlisted the help of student representatives and physician leaders to assist in recruiting additional participants. ${ }^{16}$ We obtained informed consent from all participants and gave them medical penlights as tokens of appreciation for their participation.

\section{Interviews}

To assist with generating an initial list of questions, we conducted a literature review of articles related to ethical considerations of global health experiences. Although we did not find guidelines for assessing host institution impressions and expectations, we noted expatriate involvement with clinical care, education, and research abroad, and focused our interview questions on these domains. Based on emphasis in the current literature, we also developed questions that assess host needs. ${ }^{18,20}$ We organised questions into four interview guides (one for students, interns, registrars, and faculty, respectively) and piloted them for further refinement. After informed consent, participants then underwent semistructured, one-on-one interviews. Interviewers (NP, AS, and IM) were not involved in training at the COM. The interviews were audio-recorded and transcribed verbatim.

\section{Qualitative analysis}

Transcriptions were de-identified prior to analysis and uploaded to NVivo 10 (QSR International, Doncaster, Australia), a computer software programme that supports qualitative data analysis. We used thematic analysis to conduct exploratory analyses. ${ }^{17}$ Three investigators (AS, NP, and IM) reviewed a portion of the transcripts $(7 / 46,15 \%$ each) and developed a codebook through an open coding process. We applied the codebook to subsequent transcripts independently and in duplicate (by two interviewers), with differences resolved through discussion. We reviewed interviews and wrote analytic memos that aided in the identification of themes, and we continued to conduct interviews until no new themes emerged. Once coding was completed, we reviewed codes to determine major domains and themes, and then we selected representative quotations.

\section{Results}

The 46 interviewed participants included 13 medical students, 12 interns, 10 registrars, and 11 faculty physicians. Thirty-six were participants from Queen Elizabeth Central Hospital in Blantyre and 10 were from Kamuzu Central Hospital in Lilongwe. Of the faculty and registrars, 7 were from the Department of Medicine, 4 were from Paediatrics, 3 were from Obstetrics and Gynaecology, 3 were from Ophthalmology, 3 were from Surgery, and 1 was from Anaesthesia and Intensive Care. Thirty (65\%) were male. Participants discussed a variety of themes within the following domains: perceived benefits of expatriate physicians in Malawi, perceived challenges, their past contributions, and perceived roles that expatriates should play going forward.

\section{Perceived benefits of expatriate physicians in Malawi}

Participants discussed the following benefits of expatriate physicians in Malawi: positive personality characteristics, broadened perspectives, reducing isolation, and provision of clinical care.

\section{Positive personality characteristics}

Many participants commented on the generally positive personality characteristics of expatriates. For example, students and interns described them as "helpful", "enthusiastic", "friendly", "motivational", "inspiring", "open", and "hardworking". Students specifically commented on the approachability of expatriates: "they are available and willing to help", and "they tend to empathise with you. If you're struggling, they say you can approach them." Another student commented that expatriates "know how to correct me when I am wrong in an encouraging and loving manner". A faculty physician felt like his working relationships with expatriates were less hierarchical than his Malawian relationships, referencing how "an American physician called me by my first name; it's uncommon in our culture to call an adult by his first name. That indicates equality between him and me." These characteristics led to a generally positive impression of expatriates in Malawi.

Interestingly, participants commented on why expatriates may be perceived more positively. One faculty physician noted that local Malawian physicians face barriers that may prohibit them from being as available as expatriate physicians: "Expatriate doctors have other sources of income and additional support and don't have the pressures that some of the Malawian colleagues might have for hunting around to make ends meet and doing private work." Similarly, a student commented that because Malawian physicians are busier, "they remember only the students who work really hard or that impressed them... so they will be friendly to only those students". A faculty member indicated cultural differences as a factor in this perception: "One of our cultural behaviours is to respect superiors to the extent that you fear approaching them." Another faculty member similarly said, "Malawian young people are respectful toward older people, and there will be certain things a student will not discuss or a certain way he will not approach a Malawian mentor because there is such a high respect to the elders... With expats, they feel more accepted. They feel it's easier to disagree ... it's very difficult for them to oppose the Malawian consultant if they are not of the same opinion."

\section{Broadened perspectives}

Expatriates offer diverse perspectives and clinical skills expertise, especially in teaching students varied approaches to diagnosis and management. A faculty member commented, "We think they are a great asset because they bring a unique set of skills and perspectives." Another student commented that expatriates offer expertise on diseases that are discussed less frequently in Malawi: "Learning from them, I can be exposed to certain techniques and conditions which we overlook because they are not available or not common in Malawi. I benefit from the widening of my understanding in the field." A registrar commented that some of the Malawians "might have gotten worn out by the system, so you need someone with a fresh perspective from somewhere else".

\section{Reducing isolation}

Faculty commented that expatriates help make Malawians feel less isolated. "Expatriates break up this feeling of being isolated in a poor country without any contacts to the outside world. They give the opportunity to feel connected to the 
greater medical community." Another faculty physician implied that this decreased isolation may lead to increased job satisfaction: "It's of enormous importance for the hospitals here, for the colleagues, also for the job satisfaction, that they won't feel isolated, they won't feel like, 'I'm working in one of the poorest countries of the world without any facilities, but people come and enrich our environment."'

\section{Providing clinical care}

Finally, expatriates were appreciated because they help fill the clinical needs of Malawi. One faculty member commented, "Non-Malawian medical physicians assist us by increasing the number of physicians. It's like they are lessening the burden on you." Another likened expatriate clinical responsibilities with those of Malawian physicians: "In the clinical realm they have loads of efforts, always trying to reduce disease burden, making ward rounds, seeing patients, like the Malawian physicians." A student commented that expatriates have the resources to devote to clinical care: "Some of them even sacrifice their time and resources just to help patients, maybe dip into their own pockets and even help the training. So I think that kind of heart and spirit is what we need if the current situation is to change."

\section{Perceived challenges of expatriate physicians in Malawi}

Several themes emerged regarding challenges with expatriates, including lack of understanding of culturespecific issues, poor adaptation to low-resource settings, communication issues with patients, and self-serving and exploitative intentions.

\section{Lack of understanding of culture-specific issues}

Participants noted that some expatriate physicians do not understand the cultural issues and obstacles that patients and trainees face. One registrar stated, "I can understand Malawian patients better than expats who don't have much understanding of the cultural and social issues affecting patients." Another registrar commented on the benefit of cultural similarities between herself and faculty: "Malawian physicians understand some of the challenges that I go through better because of the culture." Similarly, a student commented on the preference for a Malawian mentor due to cultural differences: "Malawian mentors are more helpful because they understand things from the ground about how Malawians think and are able to answer questions about my career."

\section{Poor adaptation to low-resource setting}

Participants commented that some expatriates have difficulty adapting to the low-resource setting in Malawi. A student shared her sentiment that expatriate physicians are too "pushy and aggressive", while others seemed to approach clinical care as more futile. For example, one registrar noted, "Some come with an attitude that 'it's just Africa, there is no good medicine." An intern similarly expressed a sense of futility by expatriates: "There are some that just think they can't practice in a poor-resource setting. If there are no resources, then there is nothing they can do... They are just visiting. So they don't care."

A faculty member commented that, "The challenge is that they have to more or less adapt to the low-resource setting here, so you find that they will be knowledgeable about a disease, but probably whatever they're mentioning, we can't do it here or we can't treat it here; so it will be good for my knowledge, but we can't actually manage patients the ways they are suggesting." Another faculty member similarly offered that expatriates may contribute to sharing knowledge but not practicality: "The expatriates can bring a lot of expertise, but the role of Malawians is to take that expertise and say how we apply it in the Malawian setting and what's appropriate, because the expatriates may not have that insight into what it means here in Malawi."

\section{Communication issues with patients}

Interviewees identified communication issues between expatriate physicians and the local population due to language and cultural barriers. One student noted, "Communication issues are a barrier when it comes to taking a history." An intern noted that "The doctor-patient interaction is not where it should be with expatriate doctors." Additionally, a faculty member commented that communication issues are a significant detriment to clinical care provided by expatriates: "It's not that she (expatriate physician) doesn't know medicine, but she lacks communication. Communication plays a big role in everything. If you can't talk the language of the people, it's like you are dumb, it's like you can't talk. You look the same."

Participants felt clinical care was smoother and rapport was stronger between physicians and patients when they communicate in the same language. One registrar said, "Malawians are more familiar with the environment, and they're able to gain rapport with patients by being able to actually speak with them, as opposed to talking through translators." Another student also stated that some patients become "scared" that they have a "serious disease" or will receive a "serious intervention" when expatriate doctors examine them, owing to miscommunication. He recalled a time when a patient left an encounter with an expatriate physician in the middle of the physical examination because the patient thought that being examined by an expatriate meant that she was going to receive an invasive procedure: "Because of her fear, she did not come back." The student felt that this misunderstanding prohibited patient care.

\section{Self-serving and exploitative intentions}

Finally, some expatriate physicians were felt to be self-serving and exploitative. A student said, "If they're here to become a professor or to be the first to find a cure to a disease, they can be remembered in history for that, but maybe they could include some of the Malawian students... For example, there was research being done on a new diagnostic test for a disease. The students involved were not from Malawi, and I understand that the technology is not from here, but there was some mumbling amongst the (Malawian) students because people were wondering, 'okay, so how come we never get opportunities to do stuff like that?" Another intern said, "Most of the expatriates are concentrated on research, so some will take advantage of the local people here, because most Malawians don't question... they can be so concentrated on their research that they forget about their actual relationships with the common people." Apart from research, students commented on the short-term stays of some expatriates: "Sometimes they are here just to get experience or whatever they need to get, and then they go back." Another student commented on how difficult it would be for a Malawian to travel elsewhere for similar experiences that expatriates receive in Malawi: "Those guys come here, do what they do, but... if we were to go there, we'd need insurance and other things. I can't just go there and do stuff, so from that perspective it's a bit one-sided." 


\section{Past contributions made by expatriates}

Several faculty participants commented on the significant contributions that expatriate physicians have made to the medical education infrastructure in Malawi. One faculty member stated, "I think their contribution in general cannot be overemphasised. Most of the college has grown from these people." In the realm of education, one noted "a positive impact from expatriates helping with the curriculum here". Another faculty member specifically recognised expatriates' contributions to training: "Most of the doctors here at some point have been trained by expat physicians, if not all, even up to now." Similarly, another said, "The non-Malawian physicians have positively affected medical education because most of them were the pioneers of the medical school, so they set the ground for the medical school, they trained the first medical students, they mentored the first medical students, and the one speaking, yes, sitting here talking, is a product of them."

\section{Perceived roles that expatriates should play}

Finally, we discussed the perceived roles that expatriates should play while abroad. To emphasise the importance of this, one faculty member stated, "I think the College of Medicine should clearly define areas where they need expatriates." Participants suggested many roles that expatriate physicians should play in Malawi, including capacity building and local empowerment, education and mentorship, and provision of opportunities for Malawian physicians and trainees to gain experience abroad.

\section{Capacity building and local empowerment}

Participants commented that expatriates should play a primary role in capacity building and empowering Malawian physician leadership. A faculty member stated, "It should be done in such a way that the expatriates train local people to eventually run the show-not just fill the gap for a year and go, because then we will be permanently dependent on others. They should train the trainers." Another faculty member reinforced this idea of encouraging Malawian leadership: "I think the leadership clearly should be in Malawian hands." An intern similarly stated, "They should also give space for Malawian physicians to develop and to be able to run the department on their own."

\section{Education and mentorship}

Expatriate physicians should continue to play roles in educating students, exchanging perspectives on varied clinical approaches, and being mentors. A faculty member stated, "They have a big role in educating Malawian students because we still do not have many Malawian physicians. Even when we have enough physicians, we need a blend of expertise and experiences." Another faculty physician similarly commented on the lack of Malawian physicians to teach trainees: "There are very few qualified Malawian doctors. If it were just Malawian physicians in the hospital, I think teaching everyone would be hard. The expatriates are helpful in the role of training many future Malawian doctors." Finally, faculty commented on the role that expatriates should continue to play as mentors to Malawian trainees: "Expatriates are role models and mentors, and they help Malawian students to network. Being their mentor means actively connecting students to people who fit their interests, providing educational materials and experiences, and being available to discuss personal issues, psychological issues, and lifestyle issues."
Offering opportunities for Malawian trainees to spend time abroad

Finally, several faculty participants expressed that expatriates should offer opportunities for Malawian trainees to have experiences abroad. One faculty member stated, "Expatriates certainly should and must create opportunities to participate in international conferences, to go abroad and do specialised courses, and meet people that have knowledge and are willing to train them. It must be a two-way road. Expats should invite students to come over and share their experiences. Part of medicine is to see how medicine is done in other parts of the world." To address the risk of worsening the brain drain phenomenon by increasing opportunities abroad, one faculty physician stated, "Opening opportunities for Malawians to maintain contacts by email, visiting, or by doing internships abroad certainly carries the risk that you invite them to leave the country. But I think this risk is quite limited because most Malawian physicians end up working back home." On the contrary, a faculty member suggested that exchange opportunities earlier in training may ultimately increase retention: "I'm strongly convinced if expats live this as a two-way road, not just coming here, seeing wonderful Malawi, travelling around, teaching a bit, but also inviting trainees to come over and share their experience with them, it could lead to job satisfaction and keep people here."

\section{Discussion}

Our study demonstrates Malawian physicians' and trainees' varied perceptions of expatriate physicians. On the positive side, expatriate physicians were felt to be approachable and hardworking. Their perspectives on clinical approaches were considered to be beneficial to trainee education, and their contributions to alleviating the physician workforce shortage and to the development of formalised medical education in Malawi have been appreciated. On the other hand, challenges with expatriate physicians were noted, including the inability to relate to patients and trainees and to adapt to low-resource settings. Some expatriates were perceived to be working in Malawi solely for their own benefit. Participants recommended that expatriates contribute to capacity building, medical student education and mentorship, and provision of opportunities for Malawians to gain experience abroad.

Best practice guidelines for expatriate physicians (for example, WEIGHT guidelines and Rules of Engagement) are primarily based on data from expatriate experiences and expert recommendations. ${ }^{9,18-20}$ They recommend that expatriates act in a culturally sensitive manner, that both host institutions and expatriates define expatriates' roles based on local needs, and that host institutions and local populations must not be exploited. However, one of the biggest limitations of these guidelines is the lack of data reflecting on the experiences of host countries. This study thus serves to help fill the need to assess the perspectives of host physicians and trainees.

Our findings can be used to help inform revisions of existing guidelines for expatriates working abroad, and they should prompt further assessment of the potential benefits and harms of global health efforts on local populations. The results emphasise the currently one-sided nature of the expatriate presence in Malawi, and suggest the need for a stronger reciprocal partnership between hosts and expatriates at various levels. At an institutional level, the findings could support the establishment of expectations that host 
institutions should have (and explicitly communicate) when developing partnerships with global health programmes, based on locally identified priorities and needs. For example, host institutions could require exchange programmes where expatriates host local physicians and trainees in exchange for their own global health experiences. As noted by one participant, global health exchange programmes could present a challenge in medical school graduate retention within Malawi. However, although many Malawian students express a desire to leave Malawi, most recent Malawian graduates were found to stay in Malawi to practice, with most working for the COM, government, and non-governmental organisations. ${ }^{10,13,21}$ Nonetheless, little is known about whether those who have experiences abroad are more likely to leave the country for work, and more research is needed on the future career endeavours of the sub-population of trainees and physicians who have had experiences abroad. Additionally, global health programmes can use our findings to optimise their roles in nurturing a clinical environment that promotes local empowerment and self-sustainability. For example, programmes could require that expatriates play stronger roles in training and capacity building. Researchers could ensure collaboration with host physicians, and clinicians could ensure that teaching and capacity building are in their core responsibilities. Host-expatriate relationships could be discontinued if efforts to collaborate and to ensure mutual benefits are not taken seriously. Finally, on an individual level, our results can assist physicians who are preparing for experiences abroad and who would like to maximise their potential to benefit both host institutions and themselves. For example, prior to their arrival, expatriates could be equipped with some basic understanding of the local language, or at the least, knowledge of medically relevant phrases, as well as local cultural views of health and healthcare.

There are some limitations to our study. First, it was conducted at a single institution at two sites, which may limit transferability to other settings. Second, the authors who played large roles in conducting interviews, coding transcripts, and analysing data were expatriates themselves and therefore could have influenced participants' responses, coding, and result interpretation. To help minimise bias, all interviews were de-identified prior to coding, and coding was performed independently.

\section{Conclusions}

Expatriate physicians play significant roles in clinical care, education, and research in low-resource settings. Guidelines acknowledge the importance of considering local population needs and attitudes when working as an expatriate; our data serve to provide further insight by highlighting the perspectives of Malawian physicians and trainees. This study, and future studies like it, can assist global health practitioners and institutions to enhance partnership building and ensure reciprocal relationships.

\section{Acknowledgements}

We thank our study participants for their time and insight.

\section{Funding}

NP's time was supported by the HRSA NRSA T32 for Primary Medical Care Research (T32HP22240).

\section{References}

1. International health residency programs [Internet]. Sterling (VA): American Medical Student Association; 2016 [cited 2016 Jan 31] Available from: http:/www.amsa.org/advocacy/action-committees/ global-health/irhs-programs/

http://dx.doi.org/10.4314/mmj.v28i2.3
2.Association of American Medical Colleges. Medical school graduation questionnaire: 2015 all schools summary report [Internet]. Washington, D.C.: Association of American Medical Colleges; 2015 [cited 2015 Sep 2]. Available from: https://www.aamc.org/download/440552/data/2015 gqallschoolssummaryreport.pdf

3. Maki J, Qualls M, White B, Kleefield S, Crone R, Vastag B, et al. Health impact assessment and short-term medical missions: A methods study to evaluate quality of care. BMC Health Serv Res. 2008;8(1):121.

4. Gupta AR, Wells CK, Horwitz RI, Bia FJ, Barry M. The International Health Program: the fifteen-year experience with Yale University's Internal Medicine Residency Program. Am J Trop Med Hyg. 1999 Dec;61(6):1019-23.

5. Miller WC, Corey GR, Lallinger GJ, Durack DT. International health and internal medicine residency training: the Duke University experience. Am J Med. 1995;99(3):291-7.

6. Ramsey AH, Haq C, Gjerde CL, Rothenberg D. Career influence of an international health experience during medical school. Fam Med. 2004;36(6):412-6.

7. Sawatsky AP, Rosenman DJ, Merry SP, McDonald FS. Eight years of the Mayo International Health Program: what an international elective adds to resident education. Mayo Clin Proc. 2010;85(8):734-41.

8. Broadhead RL, Muula AS, Grillo T, Gilles H, Dahlenburg G, Broadhead R, et al. Creating a medical school for Malawi: problems and achievements. BMJ. 2002 Aug 17;325(7360):384-7.

9. Wilson JW, Merry SP, Franz WB, Brewer TF, Saba N, Clair V, et al. Rules of engagement: the principles of underserved global health volunteerism. Am J Med. 2012 Jun;125(6):612-7.

10. Muula AS, Broadhead RL. The first decade of the Malawi College of Medicine: a critical appraisal. Trop Med Int Heal. 2001 Feb;6(2):155-9.

11. Zijlstra EE, Broadhead RL, Broadhead R, Muula A, Eastwood J, Conroy R, et al. The College of Medicine in the Republic of Malawi: towards sustainable staff development. Hum Resour Health. 2007 Dec 13;5(1):10

12. Expatriate [Internet]. Wikipedia, the free encyclopedia. 2016 [cited 2016 Jan 31]. Available from: https://en.wikipedia.org/w/index.php?titl $\mathrm{e}=$ Expatriate\&oldid $=728026747$

13. Mandeville KL, Ulaya G, Lagarde M, Gwesele L, Dzowela T, Hanson K, et al. Early career retention of Malawian medical graduates: a retrospective cohort study. Trop Med Int Heal. 2015 Jan;20(1):10614.

14. Masters of Medicine [Internet]. Blantyre: College of Medicine, University of Malawi; 2015 [cited 2015 Sep 2]. Available from: http:// www.medcol.mw/medcolnew/?page_id=381

15. Updated student enrolment. Blantyre: College of Medicine, University of Malawi; 2015.

16. Patton MQ. Qualitative research and evaluation methods: theory and practice. Thousand Oaks (CA): SAGE Publications; 2015. $832 \mathrm{p}$.

17. Crump JA, Sugarman J, Barry M, Bhan A, Gardner P, Koplan JP, et al. Ethics and best practice guidelines for training experiences in global health. Am J Trop Med Hyg. 2010 Dec 6;83(6):1178-82.

18. Crump JA, Sugarman J. Ethical considerations for short-term experiences by trainees in global health. J Am Med Assoc. 2008 Sep 24;300(12):1456-8.

19. Guest G, MacQueen KM, Namey EE. Applied thematic analysis. Thousand Oaks (CA): SAGE Publications; 2012.

20. Roberts M. Duffle bag medicine. JAMA. 2006 Apr 5;295(13):14912 .

21. Mandeville KL, Bartley T, Mipando M, Anand S, Barnighausen T, Robinson J, et al. Future career plans of Malawian medical students: a cross-sectional survey. Hum Resour Health. 2012 Dec 13;10(1):29. 